\title{
Distribution of Monocarboxylate Transporters in the Peripheral Nervous System Suggests Putative Roles in Lactate Shuttling and Myelination
}

\author{
Enric Domènech-Estévez, ${ }^{1}$ Hasna Baloui, ${ }^{1}{ }^{\circledR C}$ Cendrine Repond, ${ }^{2}$ Katia Rosafio, ${ }^{2}$ Jean-Jacques Médard, ${ }^{1}$ \\ Nicolas Tricaud, ${ }^{3}$ Luc Pellerin, ${ }^{2}$ and Roman Chrast ${ }^{1,4}$ \\ ${ }^{1}$ Departments of Medical Genetics and ${ }^{2}$ Physiology, University of Lausanne, Lausanne, CH-1005 Switzerland, ${ }^{3}$ INSERM U1051, Institut des Neurosciences \\ de Montpellier (INM), Université de Montpellier 1 and 2, 34967 Montpellier, France, and ${ }^{4}$ Department of Neuroscience and Department of Clinical \\ Neuroscience, Karolinska Institutet, 17177 Stockholm, Sweden
}

Lactate, a product of glycolysis, has been shown to play a key role in the metabolic support of neurons/axons in the CNS by both astrocytes and oligodendrocytes through monocarboxylate transporters (MCTs). Despite such importance in the CNS, little is known about MCT expression and lactate function in the PNS. Here we show that mouse MCT1, MCT2, and MCT4 are expressed in the PNS. While DRG neurons express MCT1, myelinating Schwann cells (SCs) coexpress MCT1 and MCT4 in a domain-specific fashion, mainly in regions of noncompact myelin. Interestingly, SC-specific downregulation of MCT1 expression in rat neuron/SC cocultures led to increased myelination, while its downregulation in neurons resulted in a decreased amount of neurofilament. Finally, pure rat SCs grown in the presence of lactate exhibited an increase in the level of expression of the main myelin regulator gene Krox20/Egr2 and the myelin gene P0. These data indicate that lactate homeostasis participates in the regulation of the SC myelination program and reveal that similar to CNS, PNS axon-glial metabolic interactions are most likely mediated by MCTs.

Key words: lactate; monocarboxylate transporters; peripheral nervous system; Schwann cells

\section{Introduction}

Lactate is a product of aerobic glycolysis that plays diverse physiological roles in the CNS. In addition to its well known metabolic role in sustaining synaptic function (Pellerin and Magistretti, 1994; Pellerin, 2003) and axonal activity (Tekkök et al., 2005), it has been proposed to act also both as a neurotransmitter through specific receptors (Tang et al., 2014) and as a modulator of NMDA receptor-mediated synaptic plasticity via a redox effect (Yang et al., 2014). Additionally, lactate was proposed to be used as a fuel for proper myelination by oligodendrocytes (SánchezAbarca et al., 2001). Nevertheless, one of lactate's crucial roles remains the metabolic support of CNS neurons (Lee et al., 2012). Recently, it was shown that disrupting the transfer of lactate from oligodendrocytes to axons through monocarboxylate transporter 1 (MCT1) leads to axonal damage (Lee et al., 2012). The prominence of lactate transfer from oligodendrocytes to axons is emphasized by the observation that mature oligodendrocytes

Received Aug. 23, 2014; revised Nov. 25, 2014; accepted Dec. 16, 2014.

Author contributions: E.D.-E., N.T., L.P., and R.C. designed research; E.D.-E., H.B., C.R., K.R., and J.-J.M. performed research; E.D.-E., H.B., C.R., K.R., N.T., L.P., and R.C. analyzed data; E.D.-E., N.T., L.P., and R.C. wrote the paper.

This work was supported by the University of Lausanne and the Swiss National Science Foundation grants 31003A_135735/1 (to R.C.) and 31003A_140957 (to L.P.). We also thank Dr. Dies Meijer for providing us with Krox20 antibody.

The authors declare no competing financial interests.

Correspondence should be addressed to Roman Chrast, Department of Neuroscience and Department of Clinical Neuroscience, Karolinska Institutet, Retzius väg 8, 17177 Stockholm, Sweden. E-mail: roman.chrast@ki.se. DOI:10.1523/JNEUROSCI.3534-14.2015

Copyright $\odot 2015$ the authors $\quad 0270-6474 / 15 / 354151-06 \$ 15.00 / 0$ survive and myelinate normally in the absence of mitochondrial function (Fünfschilling et al., 2012). Despite this information available for the CNS, little is known about MCT expression and lactate function in the PNS.

MCTs belong to the SLC16 superfamily (Halestrap, 2012). Four of its members (MCT1-4) are facilitated transporters for monocarboxylates, each exhibiting a different affinity for lactate and pyruvate (Halestrap, 2012). Their expression in the CNS appears to be preferentially associated with (but not always restricted to) a particular cell type, often correlating with their main metabolic phenotype. Thus, MCT1, MCT2, and MCT4 are predominantly expressed, respectively, in oligodendrocytes, neurons, and astrocytes (Lee et al., 2012), while MCT3 is found only in retinal pigment epithelial cells (Yoon et al., 1997).

Here we show that MCTs are also present in the PNS with distinct patterns of expression. In particular, we demonstrate that myelinated Schwann cells (SCs) coexpress MCT1 and MCT4 in different cellular compartments. Interestingly, by selectively downregulating MCT1 expression in either SCs or in neurons of DRG neuron/SC cocultures, we reveal its importance for both SCs' capacity to myelinate and for axonal physiology. Finally, our data from purified SCs indicate that at least part of the MCT1linked effects are mediated by lactate.

\section{Materials and Methods}

Animals. C57BL6 mice and Sprague Dawley rats obtained from Janvier Labs were used to perform immunofluorescence labelings, quantitative RT-PCR determinations, or in vitro experiments as indicated in the fol- 
A

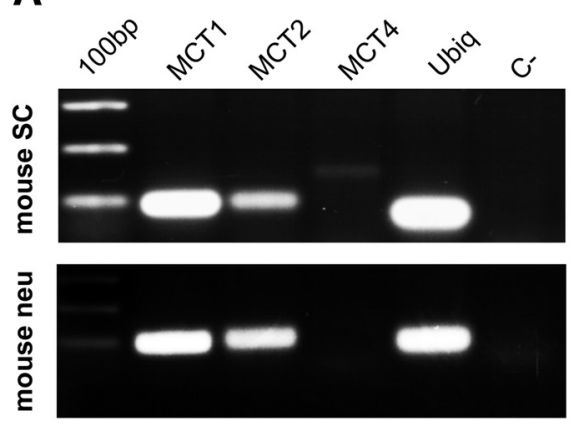

B

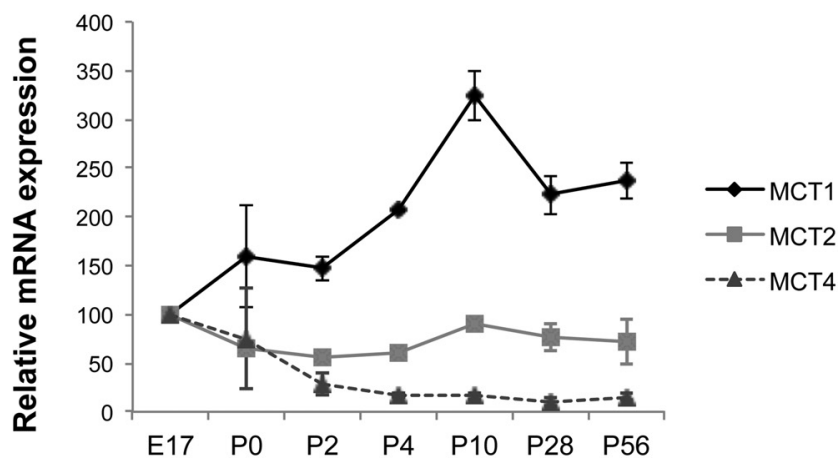

Figure 1. The expression of MCT1, MCT2, and MCT4 in the PNS. $A$, One percent agarose gel with PCR products from reverse transcription. All three transporters were detected in purified mouse SCS, and MCT1 and MCT2 were also detectable in purified mouse DRG neurons (mouse neu). Non-retrotranscripted RNA was used as a negative control (C - ). B, The expression of MCT1, MCT2, and MCT4 was analyzed by quantitative PCR, using ubiquitin-conjugating enzyme E2 as a reference. When possible (P28 and P56), the perineurium was removed from the sample and only the endoneurium was studied. Each time point corresponds to measurements performed on samples isolated from two animals and performed in triplicates. Error bars indicate SE.

lowing paragraphs. Only male mice were used for the dissections. All animal experiments were performed in accordance with the legal requirements of the University of Lausanne and the Canton of Vaud.

Cultures. Rat DRGs were obtained from E16 Sprague Dawley animals, seeded on Matrigel-coated coverslips (Corning \#356231), and cultured as described previously (Taveggia et al., 2005) to obtain pure sensory neurons. Rat SCs were obtained from P3 Sprague Dawley rat sciatic nerves and prepared as previously described (Einheber et al., 1997). Mouse SCs were isolated from E13.5 C57BL6 embryos as described previously (Stettner et al., 2013). Sodium lactate salts were obtained from Sigma-Aldrich.

shRNAs for MCT1 were obtained from Thermo Scientific. The experiments were performed with clone TRCN0000079546. Lentiviruses were produced by lipofecting $293 \mathrm{HEK}$ cells with plasmids containing PLP1, PLP2, VSVG, and the shRNA of MCT1 or scramble. Supernatants were collected $48 \mathrm{~h}$ after and used as such to infect the cells or ultracentrifuged to pellet the viruses. Infected SCs were selected with puromycin.

Purified rat SCs were lipofected with $200 \mathrm{~nm}$ scramble or siRNA MCT4 (target sequence CAGAAGCATTATCCAGATCTA) on coverslips and fixed $48 \mathrm{~h}$ later for immunofluorescence analysis.

Quantitative PCR. RNA from nerves and cells was obtained with RNeasy Lipid Tissue (Qiagen 74804) or Direct-zol RNA MiniPrep (Zymo Research R2051) kits, respectively. RNA (75-450 ng) was retrotranscripted with PrimeScript RT reagent Kit (Takara RR037A).

MCTs were detected by quantitative PCR (Maxima Hot Start Green PCR Master Mix; Thermo Scientific) and real-time PCR (FastStart Universal SYBR Green Master; Roche 10356100) following the manufacturer's indications. The $5^{\prime}-3^{\prime}$ sequences of the oligonucleotides used are as follows: rat MCT1 (Fd TATGCCGGAGGTCCTATCAG; Rev AGTTGAAAGCAAGCCCAAGA), mouse MCT1 (Fd AATGCTGCCCTGTCCTCCTA; Rev CCCAGTACGTGTATTTGTAGTCTCCAT), mouse MCT2 (Fd CAGCAACAGCGTGATAGAGCT; Rev TGGTTGCAGGTTGAATGCTAA), mouse MCT4 (Fd CAGCTTTGCCATGTTCTTCA; Rev AGCCATGAGCACCTCAAACT), mouse/rat Ubiquitin-conjugating enzyme E2 (Fd CAGCCACCAAGACTGACCAA; Rev CATTCACCAGTGCTATGAGGGA), rat Krox20 (Fd GGTGTGTGTACCATGTCCCA; Rev CCAGAGAGgagGTGGAAGTG), rat P0 (Fd TCCCTCATCCAGCCCCAGCC; Rev CTGGGAGCGCACAGCACCAT).

Immunofluorescence. For immunohistochemistry (IHC), adult mouse sciatic nerves were collected after performing a transcardial perfusion with $4 \%$ PFA. Dissected sciatic nerves were postfixed in the same solution overnight at $4^{\circ} \mathrm{C}$, cryoprotected by successive immersions in 15 and $30 \%$ sucrose in PBS, and cryosectioned at $10 \mu \mathrm{m}$ thickness. To prepare teased fibers, adult mouse sciatic nerves were dissected and immersed in $4 \%$ PFA for $30 \mathrm{~min}$ at room temperature. Nerves were teased on SuperFrost Plus slides, air dried for $2-3 \mathrm{~h}$, and kept frozen at $-80^{\circ} \mathrm{C}$ until used. DRG-SC cocultures were fixed $15 \mathrm{~min}$ in 4\% PFA, permeabilized $20 \mathrm{~min}$ in cold methanol, and processed for immunofluorescence in PBS buffer containing 5\% BSA, $1 \%$ goat serum, and $0.2 \%$ Triton X-100. The MCT1 blocking peptide CPQQNSSGDPAEEESPV was used for testing the antibody specificity in immunofluorescence.

Western blots. Cultures were collected in buffer containing 2\% SDS, 25 mм Tris, $95 \mathrm{~mm} \mathrm{NaCl}, 10 \mathrm{~mm}$ EDTA with protease, and phosphatase inhibitors (complete mini-EDTA-free tablets; Roche 11836170001, $\mathrm{Na}_{3} \mathrm{VO}_{4}, \mathrm{NaF}$ ). Fifteen to fifty micrograms of protein lysate were loaded on $10-12.5 \%$ SDS-PAGE gels, and revealed using the LI-COR Odyssey system as recommended by the company.

Antibodies. Antibodies and other markers used for IHC and Western blotting were myelin protein zero (P0; Aves Lab PZO), MBP (Millipore MAB386), Neurofilament 200 (Sigma N0142 clone 52), MCT1 (Santa Cruz Biotechnology 50325), Actin (Sigma A5316), Caspr (NeuroMab 75-001), and phalloidin 488 (Invitrogen A12379). Polyclonal antibody for Krox20 was kindly provided by Dr. Dies Meijer. Homemade polyclonal anti-MCT1 antibody was previously characterized (Pierre et al., 2000). The polyclonal anti-MCT4 antibody was obtained from Santa Cruz Biotechnology (\#SC-50329).

Statistics. One-tail Student's $t$ test was performed and results were considered significant when ${ }^{\star} p \leq 0.05$ or ${ }^{\star *} p \leq 0.001$.

\section{Results}

MCT1, MCT2, and MCT4 are present in the PNS with distinct patterns of expression

An initial analysis by RT-PCR revealed that MCT1, MCT2, and MCT4 mRNAs were detectable in cultured mouse SCs, while purified sensory neurons from mouse DRG expressed only MCT1 and MCT2 mRNAs (Fig. 1A). These results prompted us to determine the mRNA expression of MCT1, MCT2, and MCT4 in mouse sciatic nerves at different developmental times (Fig. $1 B)$. Although the three mRNAs were detected at all stages up to adulthood, MCT1 exhibited a peak of expression around the active myelination period (at P10; Fig. 1B).

The expression of MCT1 and MCT4 was further evaluated in the adult mouse PNS at the protein level. The specificity of MCT1 staining was demonstrated by neutralizing the antibody using a blocking peptide (Fig. 2A). Immunostaining on cross sections of sciatic nerve and DRG revealed that, in addition to the perineurium (Takebe et al., 2008), MCT1 is expressed in the endoneurium (Fig. $2 B$ ), and at the "capsule" of the DRG and the surface of the somas of big sensory neurons (Fig. $2 C$ ). On teased fibers prepared from 4-month-old mouse sciatic nerves, MCT1 showed a clear localization to the Schmidt-Lanterman incisures (SLIs; Fig. $2 D$ ) as confirmed by double labeling with phalloidin (Fig. 

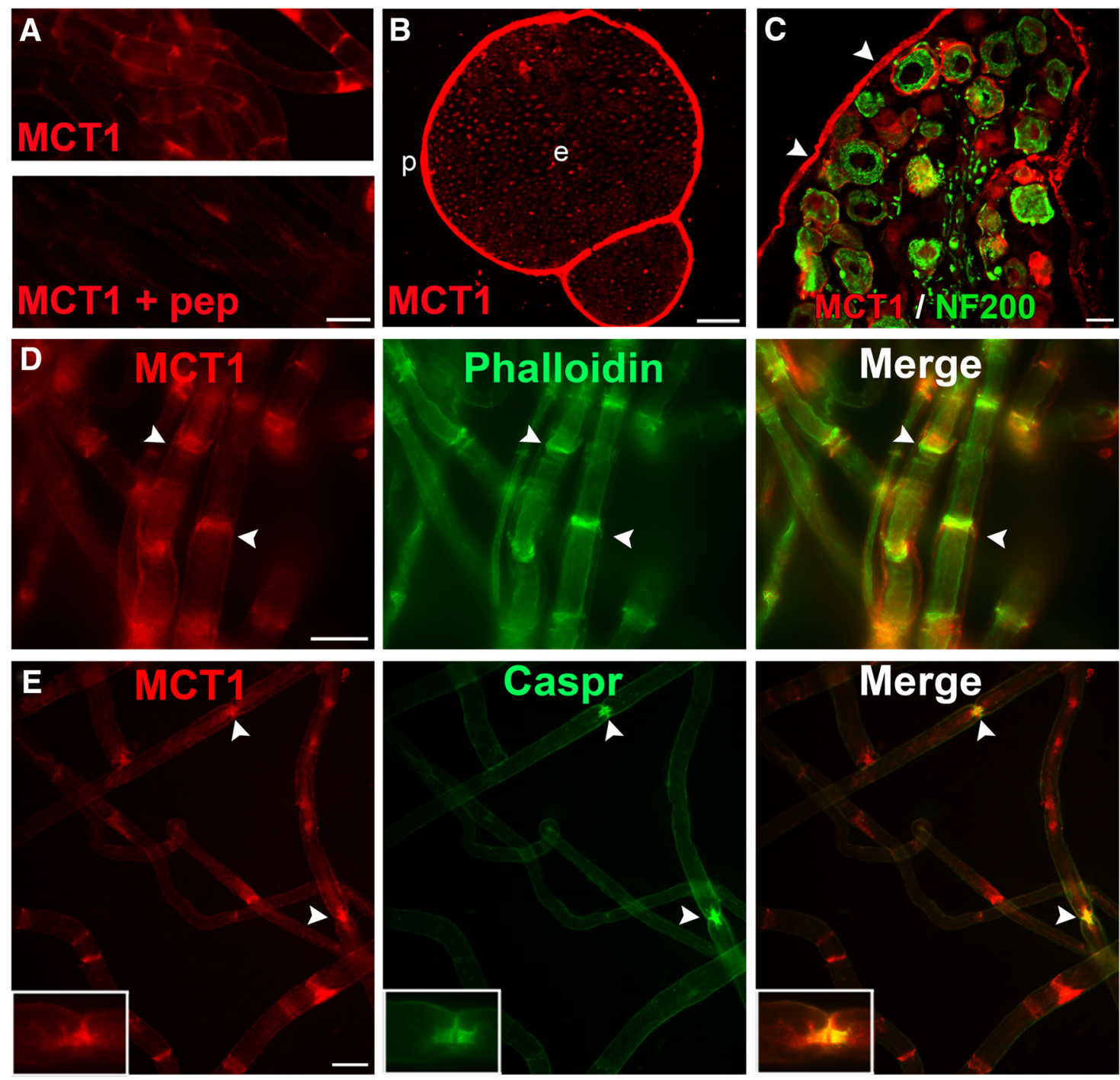

Figure 2. Localization of MCT1 in the PNS. $\boldsymbol{A}$, The MCT1 blocking peptide abrogates the MCT1 staining on teased fibers. Both images are shown with the same intensity level. $\boldsymbol{B}$, Immunostaining on cross section from adult mouse sciatic nerve shows the presence of MCT1 in both perineurium ( $p$ ) and endoneurium (e). C, Immunostaining on cross section from adult mouse DRG revealed that MCT1 is particularly enriched in the "capsule" (arrowheads) and at the surface of soma of a subset of sensory neurons. $D$, MCT1 is strongly present at the SLl, colocalizing with phalloidin (arrowheads). $\boldsymbol{E}, \mathrm{MCT1}$ colocalizes with Caspr indicating its presence in paranodal regions (arrowheads). Inserts show a magnification of the nodal and paranodal region. Scale bars: $\boldsymbol{A}, \boldsymbol{C}-\boldsymbol{E}, 20 \mu \mathrm{m} ; \boldsymbol{B}, 50 \mu \mathrm{m}$.

$2 D$, arrowhead) and at paranodal regions as visualized by double labeling with Caspr (Fig. 2E, arrowheads and inserts).

The presence of MCT4 in SCs and the specificity of the antibody were demonstrated by knocking down the transporter in cultured rat SCs (Fig. 3A). Immunostaining on cross sections of sciatic nerve revealed the presence of MCT4 in the endoneurium (Fig. 3B). On teased fibers, MCT4 was present in the SC cytoplasm as well as in an irregular mesh-like shape along the main fiber axis, suggesting its localization in the Cajal bands and outer cytoplasmic mesaxonal line (Figs. 3C,D). Double-immunofluorescence labelings from adult mouse sciatic nerve, with either NF200 or the myelin marker MBP, revealed the presence of MCT4 in the abaxonal part of the myelinating SCs (Fig. $3 E, F$, respectively, arrowheads).

\section{Reduced MCT1 expression in DRG neurons/SC cocultures} leads to decreased neurofilament expression and increased myelination

Based on the previously described role of MCT1 in oligodendrocytes (Lee et al., 2012) and its aforementioned expression in both neurons and myelinating SCs, a characterization of its putative functional role was undertaken in both PNS neurons and SCs. A cell-specific knockdown of MCT1 by lentiviral shRNAs was obtained in DRG neurons/SC cocultures and characterization of the resulting phenotype was performed for each targeted cell type (Fig. 4). The efficiency of the MCT1 knockdown was evaluated in purified SCs and neurons and found substantial (Fig. 4A). Downregulation of MCT1 in SCs resulted in an increased expression of SC-specific markers of myelination Krox20/Egr2 protein and P0 in cocultures (Fig. 4B). On the other hand, MCT1 downregulation in DRG neurons resulted in a decrease of neuronal/axonal marker NF200 expression, without affecting the myelin markers Krox 20 and P0 (Fig. 4B). The fact that no significant downregulation of MCT1 was observed in experiments shown in Figure $4 B$ reflects the mixed character of these cultures in which only one cell type is targeted by the MCT1 shRNA.

Lactate promotes transcription of myelination-related genes The increased myelination observed in vitro in mixed DRG neurons/SC cocultures with decreased levels of SC MCT1 expression raised the question of whether lactate availability in SCs could 

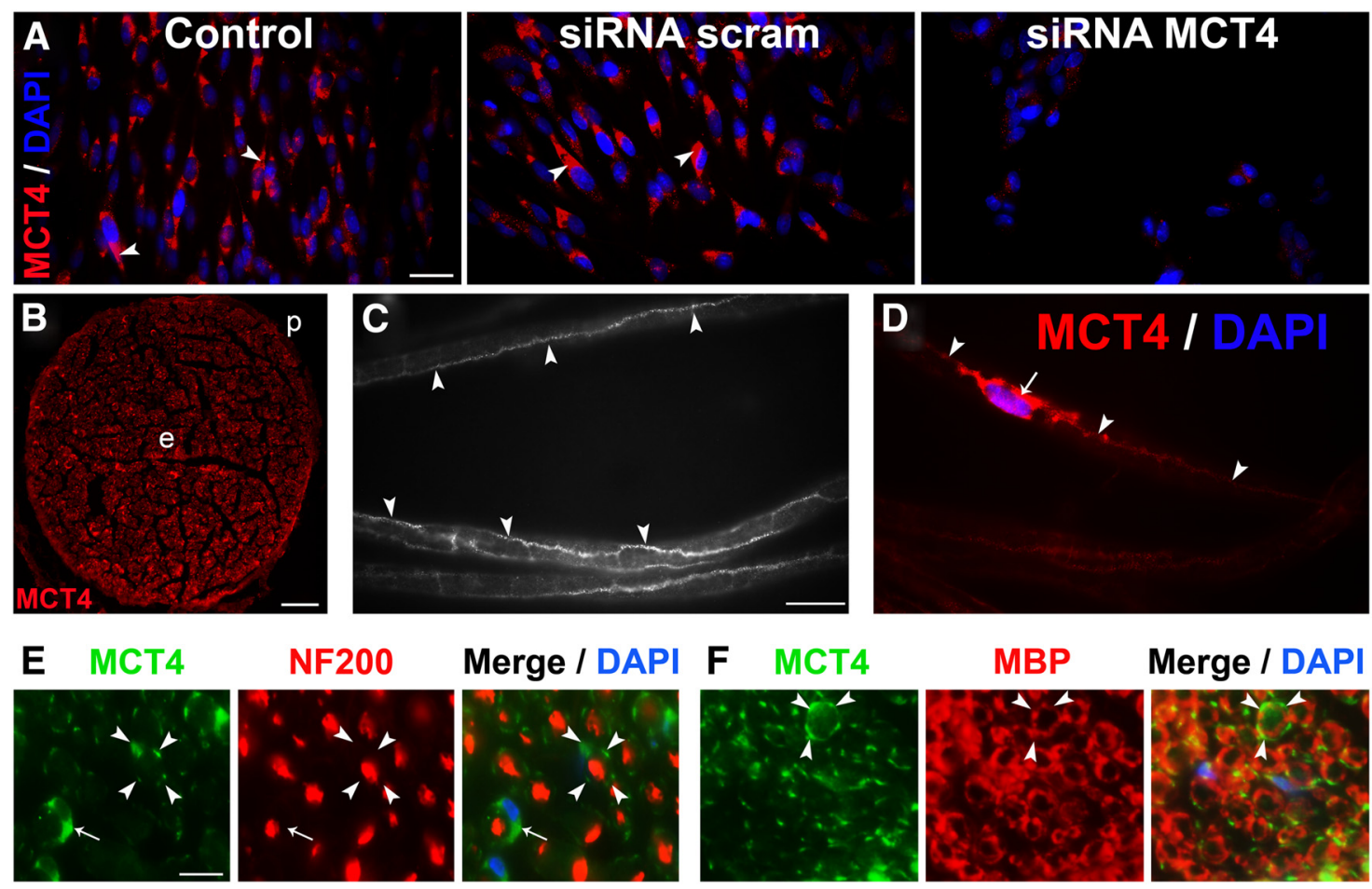

Figure 3. Localization of MCT4 in the PNS. $\boldsymbol{A}$, Cultured rat SCs were lipofected with scrambled or MCT4 siRNA. MCT4 localized to the perinuclear cytoplasm (arrowheads), and the signal was abrogated when MCT4 expression was knocked down, demonstrating both the presence of the transporter and the specificity of the antibody. $\boldsymbol{B}$, Immunostaining on cross section from adult mouse sciatic nerve revealed the presence of MCT4 in the endoneurium (e). C, D, MCT4 is expressed in myelinated teased fibers in a thin line and a mesh-like shape (arrowheads), suggesting localization in the outer cytoplasmic mesaxonal line and Cajal bands. MCT4 expression is also visible in perinuclear cytoplasm (arrow). $\boldsymbol{E}$, Cross section of sciatic nerve reveals the presence of MCT4 in the perinuclear cytoplasm (arrows) and in the most abaxonal part (arrowheads) of myelinated SCs. F, MCT4 appears as a discontinuous patchy pattern at the most abaxonal part of the fiber (arrowheads), above the myelin sheath stained by MBP. Scale bars: $\boldsymbol{A}, \boldsymbol{C}, \boldsymbol{D}, 20 \mu \mathrm{m} ; \boldsymbol{B}, 50 \mu \mathrm{m} ; \boldsymbol{E}, \boldsymbol{F}, 10 \mu \mathrm{m}$. P, perineurium.

interfere with their myelination phenotype. To assess this point, purified SCs infected with lentivirus shRNA for MCT1 or a scrambled shRNA were cultured for $2 \mathrm{~d}$ in myelinating media containing ascorbic acid and the level of mRNA expression of the myelination markers Krox 20 and $\mathrm{P} 0$ was determined by real-time PCR (Fig. 4C). As expected, MCT1 knockdown led to a substantial decrease in its own mRNA expression, which was accompanied by a significant increase in the levels of Krox 20 and P0 mRNA expression. One of the hypotheses explaining this observation could be that SCs increase the expression of myelination-related genes as a consequence of increased availability of high-energy substrates mediated by disturbed export of lactate. To determine whether lactate could be responsible for this effect, purified SCs were cultured in the same myelinating media, but in the presence of 2 mM L-lactate (Fig. 4D). Twenty-four hours after lactate addition, a significant increase in Krox20 transcription was detected. This increase was further enhanced $48 \mathrm{~h}$ after the addition of lactate. Levels of P0 mRNA were also significantly, although transiently, increased at $24 \mathrm{~h}$. These data indicate that in a lactaterich environment, obtained either by external supply or through impairment of its export, SCs enhance their Krox20 mRNA levels, likely entering into a promyelinating program.

\section{Discussion}

In contrast to the CNS, little is known about the role of lactate and MCTs in the PNS. So far only MCT1 was reported to be expressed in the sciatic nerve perineurium (Takebe et al., 2008). Here, we confirm and extend this observation by showing that in addition to MCT1, MCT2 and MCT4 are expressed in the mouse sciatic nerve. Importantly, our observations that MCT1 and MCT4 dis- play specific patterns of expression in myelinating SCs indicate that they may play particular roles in these cells.

An indication of MCTs' functional importance for the PNS comes from the temporal and spatial profile of expression. MCT1 exhibited a peak of expression around P10 and remained present at a relatively high level in consecutive time points. This could indicate an increased need for monocarboxylates, presumably lactate, in maturing PNS. Indeed, it is known that the adult PNS can release and capture lactate (Véga et al., 1998), and that such lactate, produced at least in part by the metabolism of glycogen stored in Schwann cells, can be used to sustain axonal activity (Brown et al., 2012; Evans et al., 2013).

Our data revealed that in SCs, MCT1 appears at specific locations in regions of noncompacted myelin such as SLIs and paranodal regions. SLIs are characteristic PNS myelin structures that might provide a support for metabolic shuttling allowing the exchange of metabolites between the abaxonal and adaxonal cytoplasm of myelinating SCs (Arroyo and Scherer, 2000). SLIs start to be actively formed at P5 (Poliak et al., 2002), in concordance with the observed MCT1 expression profile. In the CNS, the intercellular flux of metabolites is controlled by connexins both in astrocytes (Rouach et al., 2008) and in oligodendrocytes (Morrison et al., 2013). SLIs and paranodal regions are also rich in connexins, in particular connexin 32 ( $\mathrm{Cx} 32)$, speeding up to one million times the radial diffusion of small molecules between the abaxonal and adaxonal regions (Balice-Gordon et al., 1998). Importantly, mutations in $\mathrm{Cx} 32$ are the cause of the X-linked form of Charcot-Marie-Tooth disease, characterized by a progressive axonal impairment and demyelination (Kleopa et al., 


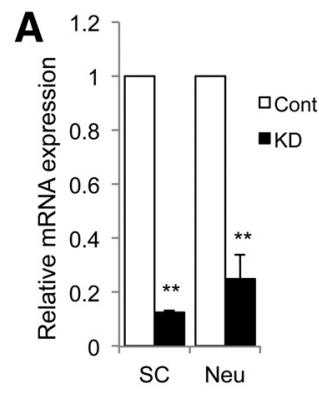

C

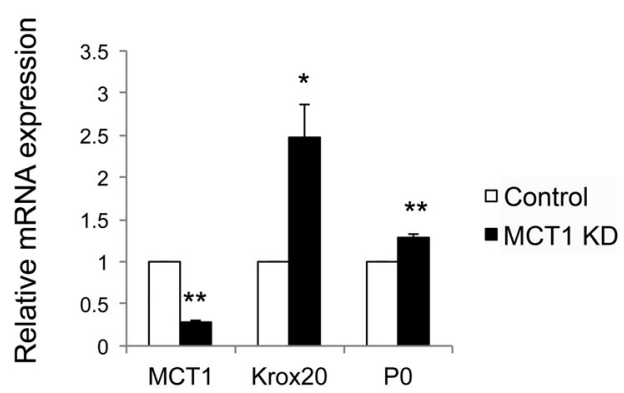

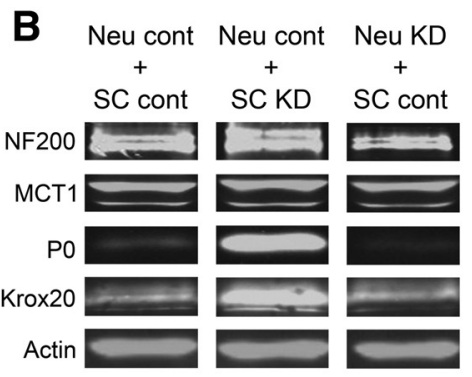

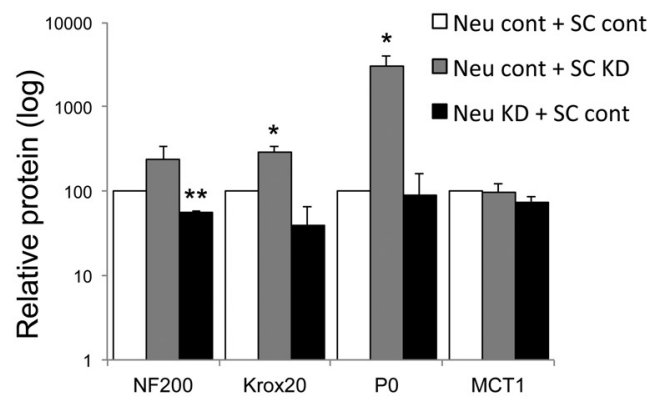

D
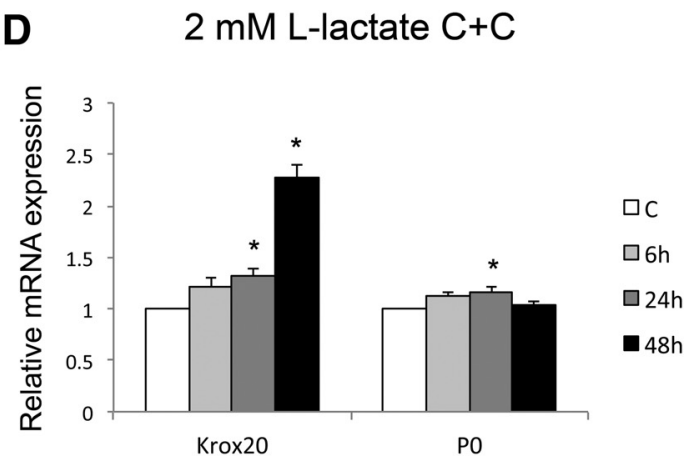

Figure 4. Downregulation of MCT1 in vitro leads to cell type-specific phenotypes and modulates the transcription of myelin-related markers in purified SCs. Rat DRG neurons (Neu) and SCs were infected with lentiviruses containing shRNA for MCT1 (KD) or scramble (cont) and selected with puromycin. The neurons and SCs were cocultured for 2 weeks in myelinating conditions. All experiments were performed in triplicates. All values refer to the scramble (controls), normalized as 1 or $100 . A, 0 \mathrm{n}$ average, 87 and $75 \%$ of MCT1 mRNA depletion was achieved in purified SCs and DRG neurons, respectively. $\boldsymbol{B}$, When MCT1 KD SCs were cocultured with control neurons, a significant increase in Krox20 and P0 protein level was revealed (Krox20 292.2 $\pm 43.63, p=0.023$; P0 $3082.34 \pm 875.49, p=0.038)$. Cocultures in which only neurons were depleted for MCT1 showed a significant reduction for NF200 (55.69 $\pm 1.5, p<0.001)$ without significant alteration in the myelin markers. The histogram illustrates the quantification of protein expression from Western blots, normalized to actin. C, Rat SCs were infected with lentivirus carrying scrambled or MCT1 shRNAs and selected in SC proliferative media in the presence of puromycin. Then, cells were switched to myelinating media containing $10 \%$ FBS and ascorbic acid, and the mRNA was extracted after $2 \mathrm{~d}$. The experiments were performed in triplicates. With $>70 \%$ of MCT1 depletion $(0.27 \pm 0.03, p<0.001)$, mRNAsfor myelin markers were increased (Krox202.48 $\pm 0.37, p=0.017 ; \mathrm{P} 01.28 \pm$ $0.03, p<0.01)$. D, Rat SCs were seeded in poly-L-lysine-coated dishes and cultured in myelinating media containing $10 \%$ FBS and ascorbic acid for $1 \mathrm{~d}$. Then 2 mm of L-lactate was added to the myelinating media and cells were harvested for mRNA extraction at indicated time points. The experiment was performed in triplicate and the values were referred to the control. Twenty-four hours after lactate addition, transcription of Krox20 was significantly increased ( $1.32 \pm 0.07 p=0.022)$, and this increase was enhanced at $48 \mathrm{~h}(2.27 \pm 0.13, p=0.005)$. The expression of P0 was also transiently increased $24 \mathrm{~h}$ after addition of lactate $(1.15 \pm 0.05, p=0.049)$, returning to control levels at $48 \mathrm{~h}(1.03 \pm 0.03, p=0.182)$.

2012). In this context, it is noteworthy that a reduction in oligodendroglial MCT1 expression seems to be involved in amyotrophic lateral sclerosis, another degenerative disease (Lee et al., 2012). Based on these data and our observations, it is tempting to speculate that metabolites flowing through monocarboxylate transporters (lactate and ketone bodies, among others) and connexins, all expressed in the noncompact myelin regions of the SC, may participate in a genuine axoglial metabolic coupling in the PNS.

We observed that when MCT1 expression is downregulated in SCs cocultured with DRG neurons, an increase in myelination was detected. Furthermore, an increase in intracellular lactate concentration, mediated by either knocking down MCT1 or by adding lactate to the ascorbic acid-containing medium, led to a transient increase in the myelin markers Krox20 and P0 in pure SCs. Interestingly, in the CNS, lactate was found to be used as fuel to achieve proper myelination (Sánchez-Abarca et al., 2001). In addition, lactate has been shown to prevent the activation of the Krox20 antagonist c-Jun (Mendler et al., 2012), and is capable of directly changing the expression of certain genes by regulating HDAC activity (Latham et al., 2012), which are key enzymes in the regulation of the transcriptional program of the SC (Jacob et al., 2011). Thus, in addition to being a fuel, lactate could behave as an instructive signal helping to coordinate myelination and metabolism related functions of SCs.

On the other hand, downregulation of neuronal MCT1 in DRG neurons/SC cocultures leads to a decrease of neurofilament expression, suggesting a metabolic stress in neurons. Energy depletion was shown to ultimately lead to axonal loss in the CNS (Lee et al., 2012), linking the metabolic coupling impairment caused by reduction in MCT1 expression with neurodegenerative diseases, as demonstrated for amyotrophic lateral sclerosis. Interestingly, recent data demonstrated that disruption of the metabolic regulator LKB1 in SCs leads to changes in nerve energy homeostasis and to axonopathy (Beirowski et al., 2014). Characterization of affected animals revealed that their nerves released an increased amount of lactate, which was shown to have an axon-protective effect. These data further suggest that lactate may play an important role in axonal support, especially under disease condition.

Myelinating SCs also express MCT4, in the perinuclear and abaxonal compartments, exhibiting a discontinuous pattern that could correlate with Cajal bands and the outer cytoplasmic mesaxonal line. The Cajal bands constitute a cytoplasmic mesh-like compartment located beneath the SC plasma membrane and are related to the proper SC elongation and nerve conduction velocity (Court et al., 2004), while the mesaxonal line is a portion of the myelinated SC cytoplasm. The presence of MCT4 at this location may indicate that those structures represent a source of important glycolytic activity and lactate production, as MCT4 is often associated with highly glycolytic phenotypes (Pierre and Pellerin, 2005).

Altogether, our data suggest that in the PNS, MCTs are present in structures in which lactate likely is produced and/or flows 
through specific cell compartments until reaching its target and where directionality comes most likely through the substrate concentration rather than from a control on the expression of a particular transporter. This system, more complex than the apparently simple yet efficient "one CNS cell type-one MCT isoform" (Lee et al., 2012), could have evolved to control the energy balance, thus optimizing the function of the two major players present in the PNS nerves: Schwann cells and axons (Samara et al., 2013).

\section{References}

Arroyo EJ, Scherer SS (2000) On the molecular architecture of myelinated fibers. Histochem Cell Biol 113:1-18. CrossRef Medline

Balice-Gordon RJ, Bone LJ, Scherer SS (1998) Functional gap junctions in the Schwann cell myelin sheath. J Cell Biol 142:1095-1104. CrossRef Medline

Beirowski B, Babetto E, Golden JP, Chen YJ, Yang K, Gross RW, Patti GJ, Milbrandt J (2014) Metabolic regulator LKB1 is crucial for Schwann cell-mediated axon maintenance. Nat Neurosci 17:1351-1361. CrossRef Medline

Brown AM, Evans RD, Black J, Ransom BR (2012) Schwann cell glycogen selectively supports myelinated axon function. Ann Neurol 72:406-418. CrossRef Medline

Court FA, Sherman DL, Pratt T, Garry EM, Ribchester RR, Cottrell DF, Fleetwood-Walker SM, Brophy PJ (2004) Restricted growth of Schwann cells lacking Cajal bands slows conduction in myelinated nerves. Nature 431:191-195. CrossRef Medline

Einheber S, Zanazzi G, Ching W, Scherer S, Milner TA, Peles E, Salzer JL (1997) The axonal membrane protein Caspr, a homologue of neurexin IV, is a component of the septate-like paranodal junctions that assemble during myelination. J Cell Biol 139:1495-1506. CrossRef Medline

Evans RD, Brown AM, Ransom BR (2013) Glycogen function in adult central and peripheral nerves. J Neurosci Res 91:1044-1049. CrossRef Medline

Fünfschilling U, Supplie LM, Mahad D, Boretius S, Saab AS, Edgar J, Brinkmann BG, Kassmann CM, Tzvetanova ID, Möbius W, Diaz F, Meijer D, Suter U, Hamprecht B, Sereda MW, Moraes CT, Frahm J, Goebbels S, Nave KA (2012) Glycolytic oligodendrocytes maintain myelin and longterm axonal integrity. Nature 485:517-521. CrossRef Medline

Halestrap AP (2012) The monocarboxylate transporter family-structure and functional characterization. IUBMB Life 64:1-9. CrossRef Medline

Jacob C, Christen CN, Pereira JA, Somandin C, Baggiolini A, Lötscher P, Ozcelik M, Tricaud N, Meijer D, Yamaguchi T, Matthias P, Suter U (2011) HDAC1 and HDAC2 control the transcriptional program of myelination and the survival of Schwann cells. Nat Neurosci 14:429-436. CrossRef Medline

Kleopa KA, Abrams CK, Scherer SS (2012) How do mutations in GJB1 cause X-linked Charcot-Marie-Tooth disease? Brain Res 1487:198-205. CrossRef Medline

Latham T, Mackay L, Sproul D, Karim M, Culley J, Harrison DJ, Hayward L, Langridge-Smith P, Gilbert N, Ramsahoye BH (2012) Lactate, a product of glycolytic metabolism, inhibits histone deacetylase activity and promotes changes in gene expression. Nucleic Acids Res 40:4794-4803. CrossRef Medline

Lee Y, Morrison BM, Li Y, Lengacher S, Farah MH, Hoffman PN, Liu Y, Tsingalia A, Jin L, Zhang PW, Pellerin L, Magistretti PJ, Rothstein JD (2012) Oligodendroglia metabolically support axons and contribute to neurodegeneration. Nature 487:443-448. CrossRef Medline

Mendler AN, Hu B, Prinz PU, Kreutz M, Gottfried E, Noessner E (2012)
Tumor lactic acidosis suppresses CTL function by inhibition of p38 and JNK/c-Jun activation. Int J Cancer 131:633-640. CrossRef Medline

Morrison BM, Lee Y, Rothstein JD (2013) Oligodendroglia: metabolic supporters of axons. Trends Cell Biol 23:644-651. CrossRef Medline

Pellerin L (2003) Lactate as a pivotal element in neuron-glia metabolic cooperation. Neurochem Int 43:331-338. CrossRef Medline

Pellerin L, Magistretti PJ (1994) Glutamate uptake into astrocytes stimulates aerobic glycolysis: a mechanism coupling neuronal activity to glucose utilization. Proc Natl Acad Sci U S A 91:10625-10629. CrossRef Medline

Pierre K, Pellerin L (2005) Monocarboxylate transporters in the central nervous system: distribution, regulation and function. J Neurochem 94:1-14. CrossRef Medline

Pierre K, Pellerin L, Debernardi R, Riederer BM, Magistretti PJ (2000) Cellspecific localization of monocarboxylate transporters, MCT1 and MCT2, in the adult mouse brain revealed by double immunohistochemical labeling and confocal microscopy. Neuroscience 100:617-627. CrossRef Medline

Poliak S, Matlis S, Ullmer C, Scherer SS, Peles E (2002) Distinct claudins and associated PDZ proteins form different autotypic tight junctions in myelinating Schwann cells. J Cell Biol 159:361-372. CrossRef Medline

Rouach N, Koulakoff A, Abudara V, Willecke K, Giaume C (2008) Astroglial metabolic networks sustain hippocampal synaptic transmission. Science 322:1551-1555. CrossRef Medline

Samara C, Poirot O, Domènech-Estévez E, Chrast R (2013) Neuronal activity in the hub of extrasynaptic Schwann cell-axon interactions. Front Cell Neurosci 7:228. CrossRef Medline

Sánchez-Abarca LI, Tabernero A, Medina JM (2001) Oligodendrocytes use lactate as a source of energy and as a precursor of lipids. Glia 36:321-329. CrossRef Medline

Stettner M, Wolffram K, Mausberg AK, Wolf C, Heikaus S, Derksen A, Dehmel T, Kieseier BC (2013) A reliable in vitro model for studying peripheral nerve myelination in mouse. J Neurosci Methods 214:69-79. CrossRef Medline

Takebe K, Nio-Kobayashi J, Takahashi-Iwanaga H, Iwanaga T (2008) Histochemical demonstration of a monocarboxylate transporter in the mouse perineurium with special reference to GLUT1. Biomed Res 29: 297-306. CrossRef Medline

Tang F, Lane S, Korsak A, Paton JF, Gourine AV, Kasparov S, Teschemacher AG (2014) Lactate-mediated glia-neuronal signalling in the mammalian brain. Nat Commun 5:3284. CrossRef Medline

Taveggia C, Zanazzi G, Petrylak A, Yano H, Rosenbluth J, Einheber S, Xu X, Esper RM, Loeb JA, Shrager P, Chao MV, Falls DL, Role L, Salzer JL (2005) Neuregulin-1 type III determines the ensheathment fate of axons. Neuron 47:681-694. CrossRef Medline

Tekkök SB, Brown AM, Westenbroek R, Pellerin L, Ransom BR (2005) Transfer of glycogen-derived lactate from astrocytes to axons via specific monocarboxylate transporters supports mouse optic nerve activity. J Neurosci Res 81:644-652. CrossRef Medline

Véga C, Poitry-Yamate CL, Jirounek P, Tsacopoulos M, Coles JA (1998) Lactate is released and taken up by isolated rabbit vagus nerve during aerobic metabolism. J Neurochem 71:330-337. CrossRef Medline

Yang J, Ruchti E, Petit JM, Jourdain P, Grenningloh G, Allaman I, Magistretti PJ (2014) Lactate promotes plasticity gene expression by potentiating NMDA signaling in neurons. Proc Natl Acad Sci U S A 111:12228-12233. CrossRef Medline

Yoon H, Fanelli A, Grollman EF, Philp NJ (1997) Identification of a unique monocarboxylate transporter (MCT3) in retinal pigment epithelium. Biochem Biophys Res Commun 234:90-94. Medline 